\title{
Article
}

\section{Twisted Waves near a Plasma Cutoff}

\author{
José Tito Mendonça * ${ }^{\mathbb{D}}$, Camilla Willim and Jorge Vieira
}

check for

Citation: Mendonça, J.T.; Willim, C.;

Vieira, J. Twisted Waves near a

Plasma Cutoff. Symmetry 2022, 14,

146. https://doi.org/10.3390/

sym14010146

Academic Editor: Alessandro Curcio

Received: 27 November 2021

Accepted: 7 January 2022

Published: 12 January 2022

Publisher's Note: MDPI stays neutral with regard to jurisdictional claims in published maps and institutional affiliations.

Copyright: (C) 2022 by the authors. Licensee MDPI, Basel, Switzerland. This article is an open access article distributed under the terms and conditions of the Creative Commons Attribution (CC BY) license (https:// creativecommons.org/licenses/by/ $4.0 /)$.
IPFN, Instituto Superior Técnico, Universidade de Lisboa, Av. Rovisco Pais 1, 1049-001 Lisboa, Portugal; camilla.willim@tecnico.ulisboa.pt (C.W.); jorge.vieira@tecnico.ulisboa.pt (J.V.)

* Correspondence: titomend@tecnico.ulisboa.pt

\begin{abstract}
This work considers twisted wave propagation in inhomogeneous and unmagnetised plasma, and discusses the wave properties in the cutoff region. The qualitative differences between twisted waves described by a single Laguerre-Gauss (LG) mode, and light springs resulting from the superposition of two or more LG modes with different frequency and helicity are studied. The peculiar properties displayed by these waves in the nonuniform plasma are discussed. The pulse envelope of a light-spring shows a contraction at reflection, which resembles that of a compressed mechanical spring. The case of normal incidence is examined, and nonlinear ponderomotive effects are discussed, using theory and simulations.
\end{abstract}

Keywords: POAM; Laguerre-Gauss; plasma; cutoff

\section{Introduction}

The problem of electromagnetic wave propagation in nonuniform plasmas, near plasma cutoff, when the wave frequency $\omega$ is close to the local electron plasma frequency $\omega_{p}$, has been considered in plasma physics for a very long time. It was first motivated by the study of radio-wave propagation in the ionosphere, as described by the classical books of Budden [1] and Ginzburg [2]. In more recent years, the problem was revisited in the context of laser-plasma interactions and laser-driven fusion research [3,4]. In this context, the mechanisms of resonant absorption [5], excitation of quasi-static magnetic fields [6], and radiation pressure effects [7] have also been considered. In these classical studies, the electromagnetic waves were described as plane waves and the transverse properties of the incident wave beam were generally ignored. Here, we return to this problem by assuming that the incident waves not only have a transverse finite dimension, but also carry a finite amount of orbital angular momentum. In other words, they are twisted waves [8].

The study of laser-matter interactions using twisted laser beams has received considerable attention in recent years [8-10]. It is well known that photon states in such twisted beams carry a finite amount of orbital angular momentum (OAM) and can be described by Laguerre-Gauss (LG) modes. If instead of vacuum we consider propagation in a plasma, the twisted photons are modified. Similarly, we can define twisted electrostatic waves, such as twisted plasmons and phonons, which become coupled to transverse electromagnetic waves through nonlinear scattering processes [11,12]. However, such processes are not of our concern here.

In a recent work, we have started exploring a new paradigm of twisted waves: the physics of light springs [13]. Although the name seems strange, it will be shown here that it is particularly well suited to describe these kind of waves near a plasma cutoff. Light springs can be seen as a special arrangement of twisted waves, which not only have a twist in the field phase, but also have a twist in the energy, or in other words, they are characterised by a twisted energy profile [14]. They are, in a sense, twisted twice. Light springs allow us to explore new radiation and acceleration phenomena. In particular, we have considered their possible use for acceleration of cylindrical and helical particle beams $[13,15]$. 
Here, we study the propagation of twisted waves and light-springs in a nonuniform plasma, and the wave field structure near a plasma cutoff. We consider an isotropic plasma, and analyse the case of normal incidence. Qualitative differences between the usual twisted waves, characterised by a single LG mode, and light springs, described by a superposition of OAM modes, are explored in the present work. These differences are due to dispersion, and vanish in a non-dispersive medium. However, in a plasma, the contrast between simple twisted waves and light-springs is clear, and somewhat surprising.

The paper is organised in the following way. In Section 2, we state the basic equations of the problem. In Section 3, we study the wave equation at normal incidence, and show how the axial and transverse solutions of the electric field can be derived. In Section 4, we discuss two different examples of twisted electromagnetic waves. The first is a simple twisted wave, as described by a single Laguerre-Gauss (LG) mode, and the second is a light spring, which results from the superposition of different LG modes with different but nearby frequencies. We illustrate the qualitative differences between these two cases with the help of the corresponding energy distributions. Finally, in Section 5, we consider the nonlinear effects which can take place in the large amplitude regime and, in Section 6, we state some conclusions.

\section{Basic Equations}

We start with the electromagnetic wave equation in an unmagnetised plasma, with a given electron density profile $n_{0}(\mathbf{r})$. Assuming that the electric field is described as a solution of the form $\mathbf{E}(\mathbf{r}, t)=\mathbf{E}(\mathbf{r}) \exp (-i \omega t)$, where $\omega$ is the wave frequency, we can write the wave equation as

$$
\nabla(\nabla \cdot \mathbf{E})-\nabla^{2} \mathbf{E}-k_{0}^{2} \epsilon(\omega, z) \mathbf{E}=0,
$$

where $k_{0}^{2}=\omega^{2} / c^{2}$. In a cold plasma, we can use the following dielectric function

$$
\epsilon(\omega, z)=1-\frac{\omega_{p}^{2}(z)}{\omega^{2}}, \quad \omega_{p}^{2}(z)=\frac{e^{2} n_{0}(z)}{\epsilon_{0} m} .
$$

These equations are valid when the electron density varies over a scale much larger than the oscillation amplitude of the electrons in the wave field. They will eventually break down in the strongly nonlinear regime. Notice that the plasma frequency $\omega_{p}$ depends on the equilibrium density profile $n_{0}(z)$. The quantity $(\nabla \cdot \mathbf{E})$ can be retrieved from Maxwell's equations. Using

$$
\nabla \times \mathbf{H}=-i \omega \epsilon(\omega, z) \mathbf{E},
$$

where $\mathbf{H}$ is the wave magnetic field, and taking the divergence, we obtain

$$
(\nabla \cdot \mathbf{E})=-\mathbf{E} \cdot \nabla \ln \epsilon(\omega, z) .
$$

This equation shows that the longitudinal component of the electric field (in the direction of wave propagation) can couple with the plasma inhomogeneity. Given the definition of $\epsilon(\omega, z)$, we can easily obtain

$$
\nabla \ln \epsilon(\omega, z)=-\frac{1}{\epsilon(\omega, z)} \frac{\omega_{p}^{2}(z)}{\omega^{2}} \nabla \ln n_{0}(z) .
$$

Equations (1) and (4) are the starting point of our model. 


\section{Normal Incidence}

We assume propagation along the $z$-axis, and a density gradient parallel to the direction of propagation, $\nabla n_{0} \| \mathbf{e}_{z}$. The field in this case is transverse, and we have $(\nabla \cdot \mathbf{E})=0$. The wave Equation (1) can then be replaced by

$$
\left(\nabla_{\perp}^{2}+\frac{\partial^{2}}{\partial z^{2}}\right) \mathbf{E}+k_{0}^{2} \epsilon(\omega, z) \mathbf{E}=0,
$$

For twisted waves, it is useful to assume an electric field solution of the form

$$
\mathbf{E}(\mathbf{r})=A(z) R\left(\mathbf{r}_{\perp}, z\right) \mathbf{e}_{\omega},
$$

where $A(z)$ describes the field evolution along the direction of propagation, and $\mathbf{e}_{\omega}=\mathbf{E} / E$ is the unit polarisation vector. The transverse form function, $R\left(\mathbf{r}_{\perp}, z\right)$, to be specified later, can be assumed to weakly depend on $z$, such that

$$
\left|\frac{1}{R} \frac{\partial R}{\partial z}\right| \ll\left|\frac{1}{A} \frac{\partial A}{\partial z}\right| .
$$

Replacing this in the wave Equation (6), we obtain

$$
\left[A \nabla_{\perp}^{2} R+2 \frac{\partial A}{\partial z} \frac{\partial R}{\partial z}\right]+R\left[\frac{\partial^{2} A}{\partial z^{2}}+\frac{\omega^{2}}{c^{2}} \epsilon(\omega, z) A\right]=0 .
$$

This equation is satisfied if the axial and radial field functions simultaneously satisfy the following two separate equations

$$
\left[\frac{\partial^{2}}{\partial z^{2}}+k_{0}^{2} \epsilon(\omega, z)\right] A=0,
$$

and

$$
\left[\nabla_{\perp}^{2}+2\left(\frac{1}{A} \frac{\partial A}{\partial z}\right) \frac{\partial}{\partial z}\right] R=0 .
$$

Let us first concentrate on the axial wave Equation (10). Far away from the plasma cutoff, $z=z_{0}$, defined by the condition $\epsilon\left(\omega, z_{0}\right)=0$, or equivalently by $\omega=\omega_{p}\left(z_{0}\right)$, this equation can easily be solved assuming a solution of the form

$$
A(z)=A_{0} \exp [i \phi(z)]
$$

where $A_{0}$ is a constant amplitude, and the phase $\phi(z)$ satisfies the equation

$$
i \frac{d^{2} \phi}{d z^{2}}-\left(\frac{d \phi}{d z}\right)^{2}+k^{2}(z)=0,
$$

where the wavenumber satisfies the (local) dispersion relation

$$
k(z)=\frac{\omega}{c} N(z), \quad N(z)=\sqrt{\epsilon(\omega, z) .}
$$

Here, $N(z)$ is the refractive index. We can approximately solve the phase equation using an iterative method, where we start by neglecting the second derivative. The result is well known, and can be stated as

$$
\phi(z)= \pm \frac{\omega}{c} \int N(z) d z+i \ln \left(N^{1 / 2}\right) .
$$


This then leads to the wave solution

$$
A(z)=\sum_{ \pm} A_{ \pm} \frac{1}{\sqrt{N}} \exp \left[ \pm i \frac{\omega}{c} \int N(z) d z\right]
$$

where $A_{ \pm}$represents the amplitude of the incident and reflected waves. Replacing this in the axial wave Equation (10), we verify that this solution is only valid if the following inequality is satisfied

$$
\left[\frac{3}{4}\left(\frac{1}{N^{2}} \frac{d N}{d z}\right)^{2}-\frac{1}{2 N^{3}} \frac{d^{2} N}{d z^{2}}\right] \ll \frac{\omega^{2}}{c^{2}} .
$$

It is obvious that, near the plasma cutoff, when the wavenumber and the refractive index tend to zero, $N\left(z \rightarrow z_{0}\right) \rightarrow 0$, this inequality will eventually break down. This means that the wave solution in this critical region needs a more adequate description. For that purpose, we linearise the density profile around the critical point, and write

$$
\epsilon(\omega, z)=a\left(z-z_{0}\right), \quad a=\left(\frac{1}{n_{0}} \frac{d n_{0}}{d z}\right)_{z=z_{0}} .
$$

Here, the quantity a determines the density scale-length. Equation (10) can then be replaced by

$$
\frac{\partial^{2} A}{\partial z^{2}}+\frac{\omega^{2}}{c^{2}} a\left(z-z_{0}\right) A=0 .
$$

Using a new variable

$$
\zeta=\left(\frac{\omega^{2}}{c^{2}} a\right)^{1 / 3}\left(z-z_{0}\right),
$$

this can be written in the standard form

$$
\frac{\partial^{2} A}{\partial \zeta^{2}}-\zeta A=0
$$

This is the well-known Airy equation, which can be solved as [16]

$$
A(\zeta) \equiv A i(\zeta)=\frac{3}{\pi} \int_{0}^{\infty} \cos \left(\frac{1}{3} t^{3}+\zeta t\right) d t,
$$

There is a second independent solution, $B i(\zeta)$, which diverges at infinity and is ignored. Far away from cutoff $z=z_{0}$, we can use the asymptotic form of the Airy function $\operatorname{Ai}(z)$, given by

$$
A i(\zeta) \simeq \frac{1}{\pi}|\zeta|^{-1 / 4} \sin \left(\frac{2}{3}|\zeta|^{3 / 2}+\frac{\pi}{4}\right)
$$

This allows us to write the field amplitude (22) in the form of a standing wave, as in Equation (16), with $\left|A_{+}\right|=\left|A_{-}\right|$. On the other hand, in the evanescent region, we simply have

$$
A(\zeta) \simeq \frac{1}{2 \sqrt{\pi}}|\zeta|^{-1 / 4} \exp \left(-\frac{2}{3}|\zeta|^{3 / 2}\right)
$$

This completes our discussion on the axial evolution of the wave electric field, which follows from the well-known solutions for an incident plane wave.

\section{Twisted Modes}

Let us now consider the transverse beam structure, as described by Equation (11). Using $A(z)$, as given by the above asymptotic solutions, we can write it in the usual paraxial form, as

$$
\left[\nabla_{\perp}^{2}+2 k(z) \frac{\partial}{\partial z}\right] R=0
$$


The general solution of this equation can be represented as a superposition of LaguerreGauss (LG) functions, $F_{\ell p}$, such that

$$
R\left(\mathbf{r}_{\perp}, z\right)=\sum_{\ell p} R_{\ell p} F_{\ell p}\left(\mathbf{r}_{\perp}, z\right)
$$

where the constants $R_{\ell p}$ are the mode amplitudes. In cylindrical coordinates, $\mathbf{r}_{\perp} \equiv(r, \theta)$, we have

$$
F_{\ell, p}(r, \theta)=C_{\ell p} X^{|\ell|} L_{p}^{|\ell|}(X) \exp \left(i \ell \theta-\frac{X}{2}\right),
$$

where the functions $L_{p}^{|\ell|}(X)$ are the associated Laguerre polynomials, the variable $X=(r / w)^{2}$ is the square of the radial coordinate normalised to the beam waist $w$, and $C_{\ell p}$ are appropriate normalisation constants. We see that the LG functions depend on the poloidal coordinate as $F_{\ell p} \propto \exp (i \ell \theta)$, where $\ell$ is the azimuthal quantum number. In the cutoff region, the wavenumber $k(z)$ can be linearised, and the wave amplitude evolves according to the Airy function $\operatorname{Ai}(z)$. We assume that the transverse shape of the pulse is not significantly modified, $\partial R / \partial z \simeq 0$, and that the same LG solutions still hold. This is valid if the cutoff region is much shorter than Rayleigh length of the beam, which is a plausible assumption for a wide variety of experimental conditions. This means that, for a twisted incident beam with a single LG mode such that $R_{\ell p}=A_{0} \delta_{\ell \ell_{0}} \delta_{p p_{0}}$, the following field solution can be used

$$
E(\mathbf{r})=A_{0} F_{\ell_{0} p_{0}}(\mathbf{r}, z) A i(z) .
$$

The resulting energy distribution is represented in Figure 1. We can see that, in the cutoff region, the energy is represented by a sequence of concentric tori, with increasing width, stopping at the cutoff. This configuration results from an increasing value of the wavelength, and a consequent decrease of the local wavenumber $k(z)$. Such features are in clear contrast with the case of light springs, to be discussed next.

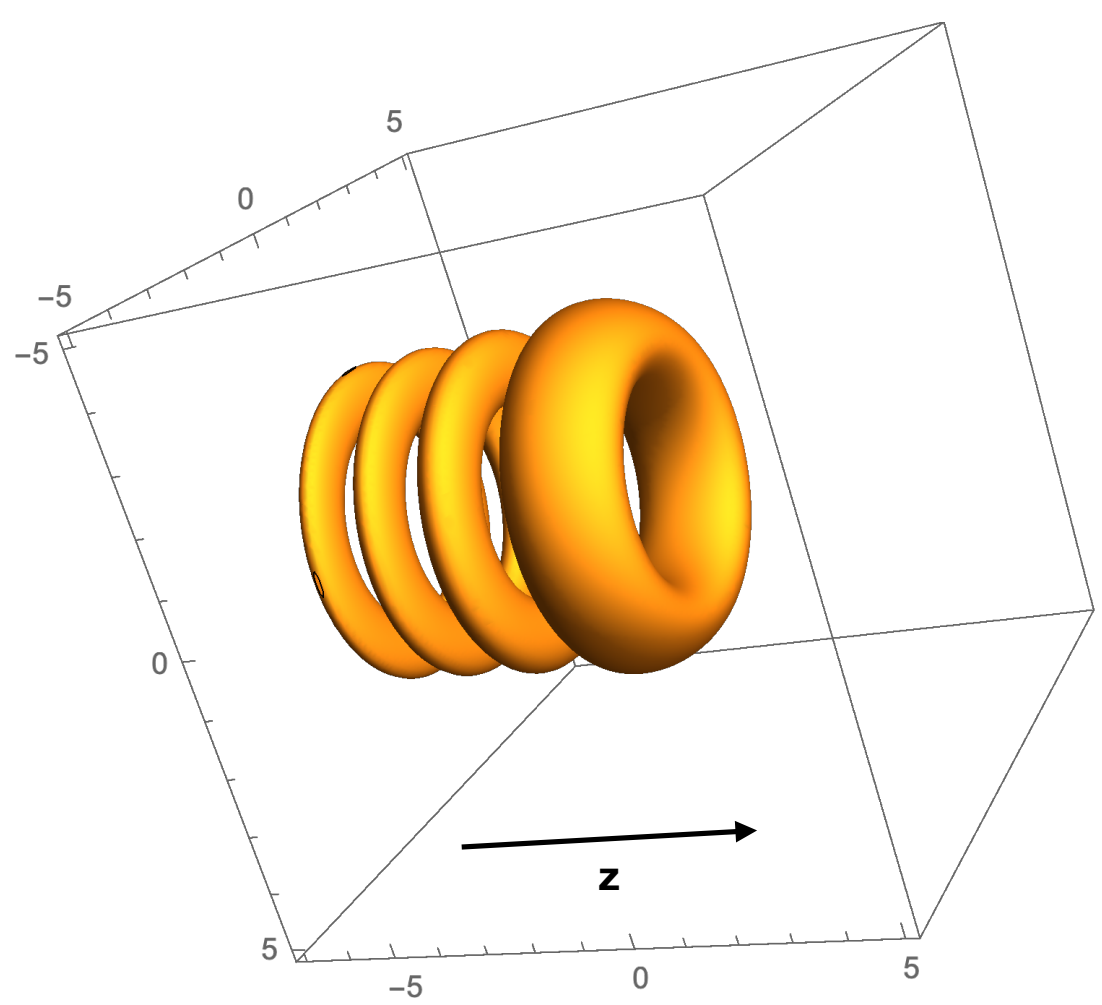

Figure 1. Energy density $\left|E\left(\mathbf{r}_{\perp}, z\right)\right|^{2}$ of a twisted wave at plasma cutoff, as described by a single LG mode $\ell=1, p=0$. 
We define light spring a superposition of LG modes with different frequencies, $\omega_{\ell}$, where the frequency difference between the consecutive azimuthal modes is constant, $\Delta \omega=\omega_{\ell}-\omega_{\ell \pm 1}$. This difference is assumed to be very small with respect to the wave and plasma frequencies, $|\Delta \omega| \ll\left(\omega_{\ell}, \omega_{p}\right)$. Let us consider the case with three modes, $\ell=\left(\ell_{0}, \ell_{0} \pm 1\right)$, and the same radial mode number $p=p_{0}$. The mode structure function (26) will become

$$
R\left(\mathbf{r}_{\perp}, z\right)=e^{i \ell_{0} \theta} \sum_{\ell=\ell_{0}, \ell_{0} \pm 1} R_{l}\left|F_{\ell p_{0}}\left(\mathbf{r}_{\perp}, z\right)\right| e^{i \Delta \ell \theta},
$$

with $\Delta \ell=\ell-\ell_{0}$. For simplicity, we ignore contributions from the Gouy phase, which could be easily included in the analysis, without significantly changing the final results. Furthermore, the cutoff positions, $z_{c}$, for each mode will be different and will depend on $\ell, z_{c}(\ell)=z_{\ell}$. We can then define, for the different modes in the cutoff region, the space variables

$$
\zeta_{\ell}=\left(\frac{\omega^{2}}{c^{2}} a\right)^{1 / 3}\left(z-z_{\ell}\right)
$$

More importantly, the frequency difference $\Delta \omega$ will imply a wavenumber difference $\Delta k=k_{0}-k_{\ell}$ which, in normalised units, is given by

$$
\Delta \kappa \equiv \frac{c \Delta k}{\omega_{p}}=\left[\frac{\omega_{0}}{\omega_{p}(z)}\right]^{2} \frac{\Delta \omega}{\omega_{0}} \frac{1}{\sqrt{\left[\omega_{0} / \omega_{p}(z)\right]^{2}-1}} .
$$

This shows that $\Delta \kappa \rightarrow \infty$, and $\kappa_{\ell} \rightarrow 0$, when the waves approach cutoff, $z \rightarrow z_{\ell}$. This qualitative difference, between the mode wavenumber, which tends to zero, and the difference between wavenumbers inside the spring, which tends to infinity, can be called the light-spring effect and leads to a markedly different behaviour at cutoff between a single LG mode and a light spring. This is illustrated in Figure 2, which compares the variation of $\Delta \kappa$ and $\kappa$ with $\omega_{p}$, and therefore with position, and Figure 3, where the energy distribution of a light spring is shown for different values of $\omega_{0} / \omega_{p}$.

We can see that the light-spring energy undergoes compression near the cutoff, during pulse reflection, and a short pulse behaves as a material spring which is released after reflection. This spring compression and decompression near the cutoff is confirmed by numerical simulations, as shown in Figure 4. The three-dimensional numerical simulations were performed using the particle-in-cell code OSIRIS $[17,18]$. The simulation window is cubic. The dimension of the cube edge is $200 \mathrm{c} / \omega_{p}$. The simulation window is divided into 1000 cells in the longitudinal $\left(x_{1}\right)$ direction and into $500 \times 500$ cells in the transverse $\left(x_{2}\right.$ and $\left.x_{3}\right)$ directions. Each cell initially contains 2 particles. The light-spring laser is a combination of two Laguerre-Gaussian modes with azimuthal indexes $\ell_{1}=1$ and $\ell_{2}=2$. The frequency of the second mode is $\omega_{2}=1.25 \omega_{1}$. In addition, the spot size for both modes is $w_{0}=30 c / \omega_{p}$. Both lasers focus at $x_{1}=100 c / \omega_{p}$. The temporal profile of each mode consists of three sections: (i) the fields rise until they reach their maximum values over a distance of $24 c / \omega_{p}$, (ii) the fields are then flat over $40 c / \omega_{p}$, and (iii) they fall back to zero over $24 c / \omega_{p}$. The peak laser normalised vector potential is $a_{1}=a_{2}=0.0428$. The plasma density profile increases linearly from $x_{1}=[110-158] c / \omega_{p}$ according to $n / n_{c}=\alpha\left(x_{1}-110\right)$, where $\alpha=1 / 16$, and $n / n_{c}=0$ otherwise. The plasma density is normalised to the critical density $n_{c}=\epsilon_{0} m_{e} \omega_{1}^{2} / e^{2}$. The critical layer for the lower frequency laser mode is thus at $x_{1}=126 c / \omega_{p}$. For the higher frequency mode is at $x_{1}=135 c / \omega_{p}$. The main difference between the two figures is that Figure 3 only shows the energy envelope, whereas in Figure 4 we can also see the fast varying electric field oscillations (blue for positive values, and red for negative values). Figure 4 shows the critical layer for the higher frequency laser mode only. Figure 4c shows that the light spring shape is slightly elongated after reflection, as a result of the laser frequency dependent reflection positions. Both figures demonstrate the spring effect at the cutoff. 


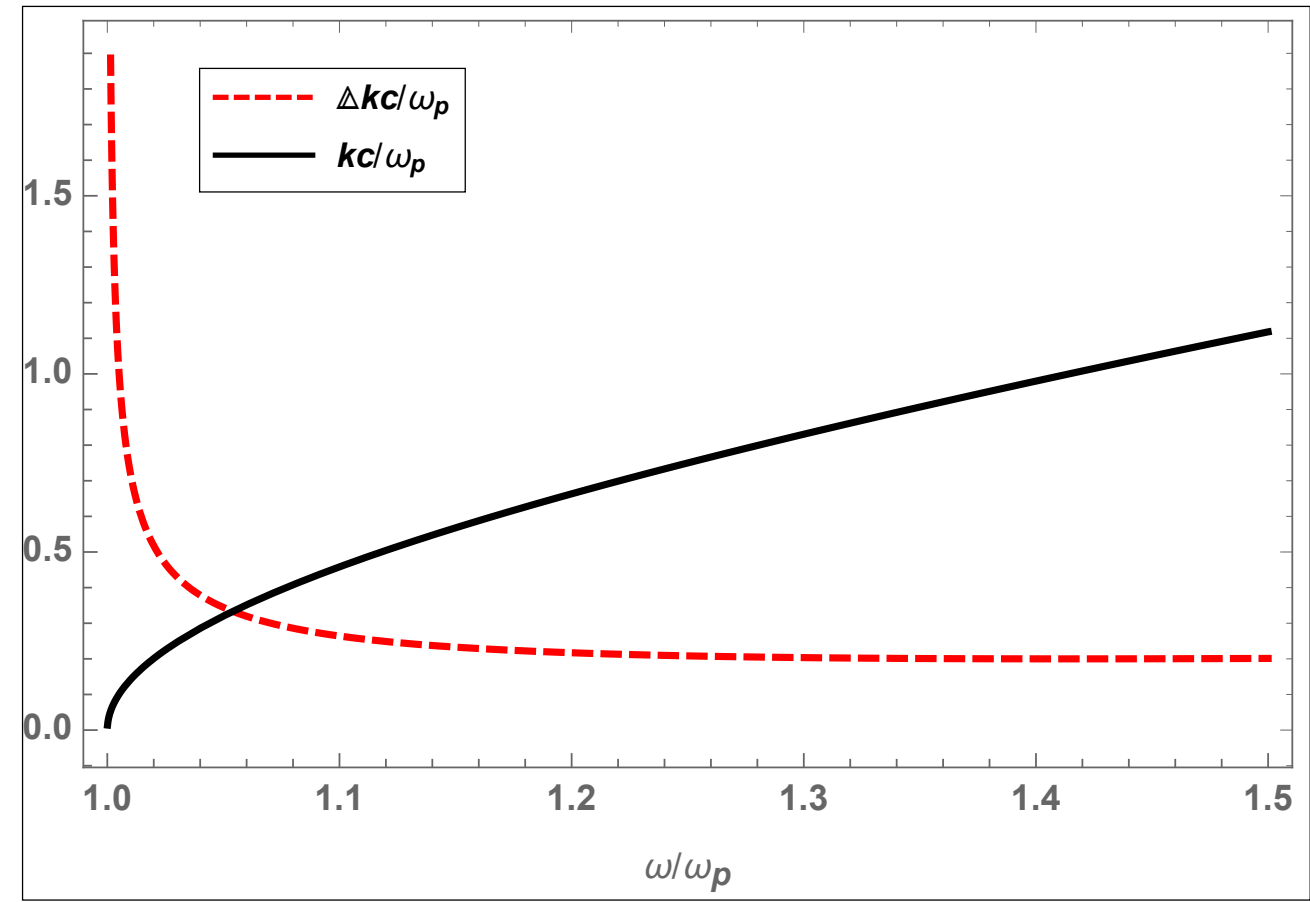

Figure 2. Normalized wavenumber of a single mode, $k c / \omega_{p}$, and difference between two modes in a light spring, $\Delta k c / \omega_{p}$, as a function of the ratio $\omega / \omega_{p}$. Near cutoff, $k \rightarrow 0$ and $\Delta k \rightarrow \infty$, as $\omega / \omega_{p} \rightarrow 1$.

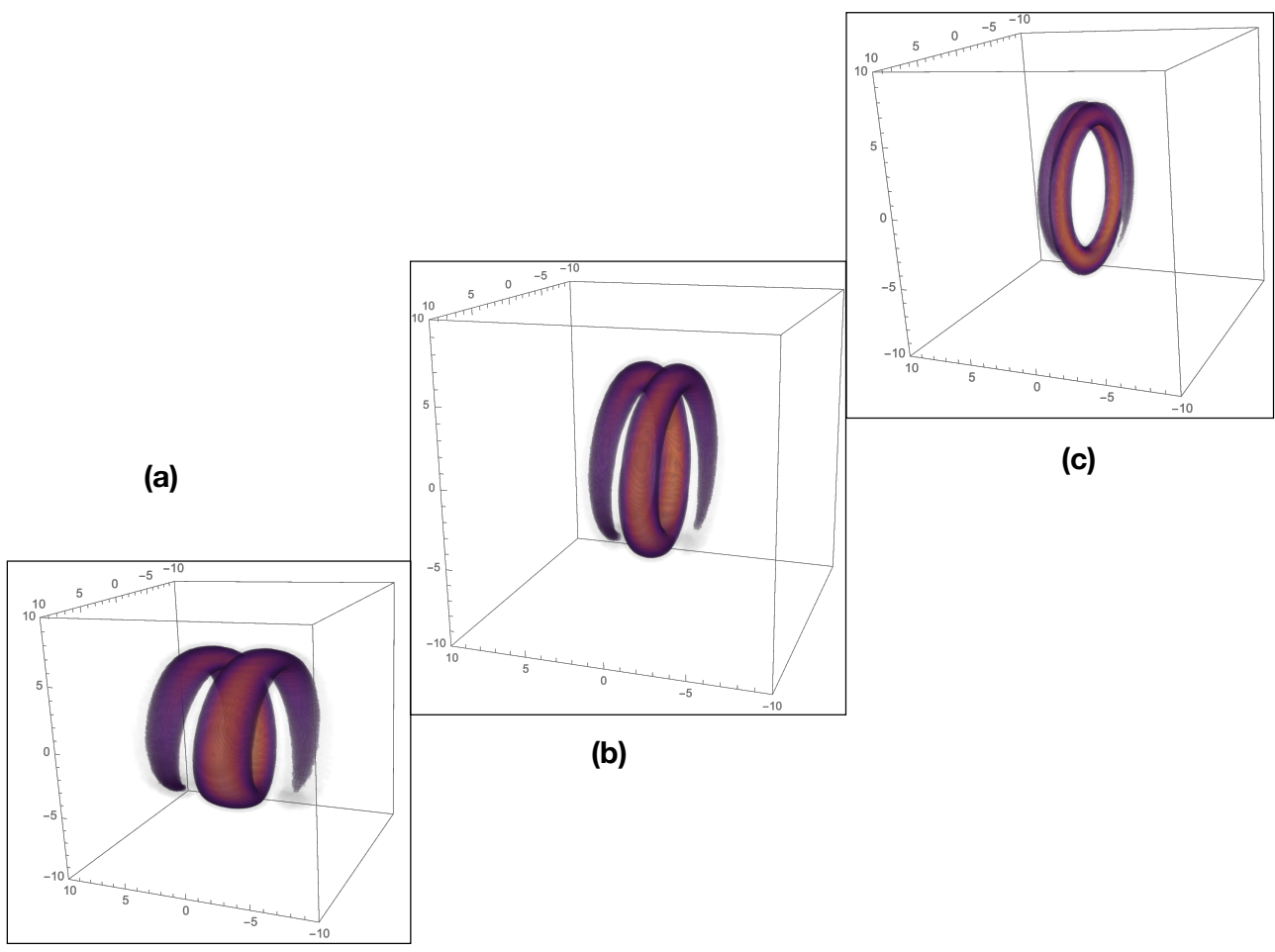

Figure 3. Energy density envelope $\left|E\left(\mathbf{r}_{\perp}, z\right)\right|^{2}$ of a light-spring approaching plasma cutoff, as described by a superposition of two LG modes with $\ell=6, p=0$ and $\ell=7, p=0$ : (a) far from the cutoff position, at $\omega=4 \omega_{p}(z)$; (b) closer to cutoff, at $\omega=2 \omega_{p}(z)$; and (c) very close to cutoff, at $\omega=1.1 \omega_{p}(z)$. 


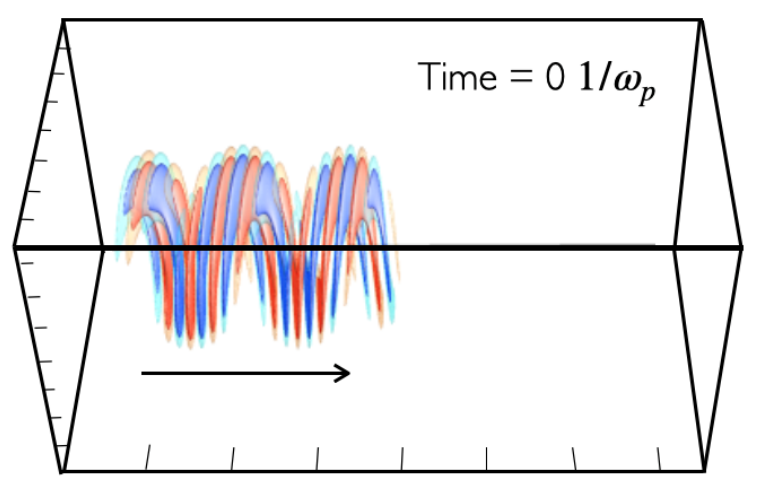

a)

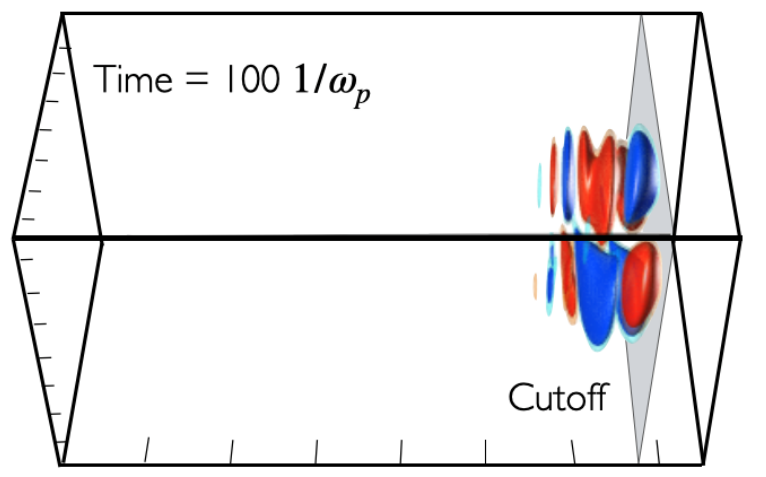

b)

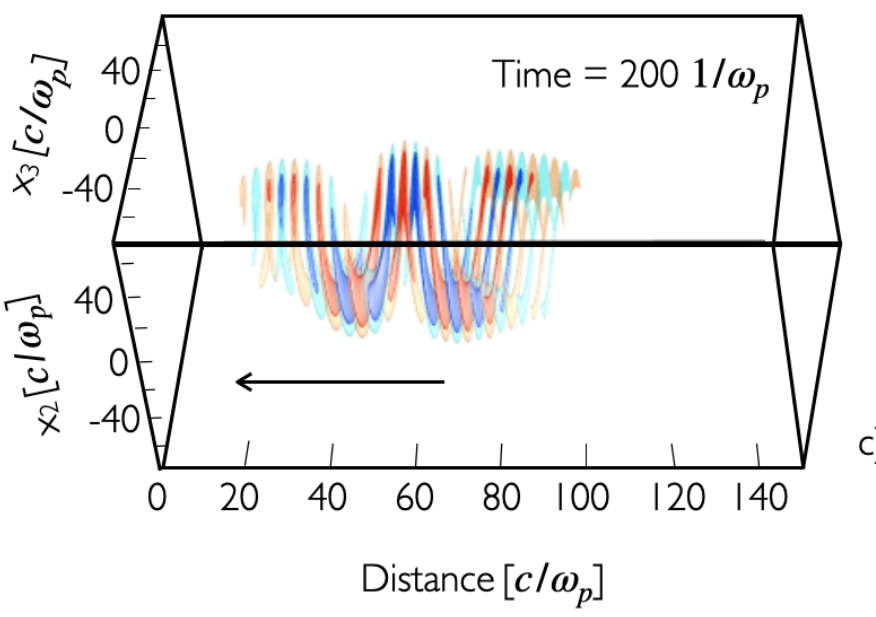

Figure 4. Simulation of a light spring in the linear regime, propagating at normal incidence near the plasma cutoff, at different computation times: (a) before reflection, $\omega_{p} t=0,(\mathbf{b})$ at cutoff, $\omega_{p} t=100$, and (c) after reflection, $\omega_{p} t=200$. The OSIRIS code was used. This shows a spring effect, confirming the analytical predictions.

\section{Nonlinear Effects}

Here, we re-examine the plasma cutoff for lasers with relativistic intensities, such that nonlinear effects associated with the ponderomotive pressure become relevant. For this purpose, we go back to Equation (1), where the electron current $\mathbf{J}=-e n \mathbf{v}$ is rewritten as

$$
\mathbf{J}=-e n c \mathbf{u} / \gamma, \quad \gamma=\sqrt{1+u^{2}},
$$


where $\mathbf{u}$ is the normalised covariant velocity, and $\gamma$ the electron relativistic factor. In order to determine this current we need to solve the relativistic momentum equation. At relativistic intensities, this equation can be approximately written as (see, for instance, [19])

$$
\frac{\partial \mathbf{u}}{\partial t}=-\frac{e \mathbf{E}}{m c}-\frac{e^{2}}{2 \gamma m^{2} c} \nabla|\mathbf{E}|^{2} .
$$

The last term in this equation is the ponderomotive force, which contains slow components with frequency much lower than the incident laser frequency. In the case of a light spring, assumed with field components of the same amplitude $E_{0}$, the module square of the total electric field is

$$
|E|^{2}=W_{0}+\left[W_{+} \exp (i \Delta \ell \theta-i \Delta \omega t)+W_{-} \exp (-i \Delta \ell \theta+i \Delta \omega t)\right],
$$

where the static and oscillating terms are determined by the quantities $W_{0}$ and $W_{ \pm}$, as given by

$$
W_{0}=\sum_{\ell}^{\ell_{0}, \ell_{0} \pm 1} R_{\ell}^{2}\left|F_{\ell p}\right|^{2}\left|E_{0}\right|^{2}, \quad W_{ \pm}=R_{\ell_{0}} R_{\ell_{0} \pm 1}\left|F_{\ell_{0} p} F_{\ell_{0} \pm 1, p}\right|\left|E_{0}\right|^{2} .
$$

Notice that the quantities $F_{\ell p}$ depend on transverse and axial coordinates, as defined above. From this, it becomes clear that the resulting electron current will have three different components, which are associated with three different nonlinear effects. First, the current determined by the linear force in Equation (33) becomes nonlinear due to the relativistic factor, and leads to a small displacement of the cutoff position, which is now defined by $\omega=\omega_{p 0} / \gamma_{0}$, where $\gamma_{0}$ is the local mean value of $\gamma$. This is the well known self-induced transparency due to a decrease of the electron effective mass. Second, the static force proportional to $W_{0}$ can be responsible for a deformation of the density profile $n(\mathbf{r})$ close to the cutoff position, a process usually associated with hole boring. In order to describe it properly we would need to include an electrostatic potential created by the static pressure, which could drive ion acceleration.Third, the term driven by $W_{ \pm}$oscillates at the frequency $\Delta \omega$ and can eventually couple to low-frequency electrostatic oscillations.

Therefore, at relativistic laser intensities, we can observe at least three distinct nonlinear processes: relativistic self-induced transparency, hole boring and low-frequency oscillations. Notice that this last process is exclusive of light springs and that the first two are also present for purely Gaussian beams and for plane waves. To these processes, we should also add particle heating which is not considered here. Figure 5 shows evidences of some of these processes from additional three-dimensional simulations using OSIRIS. Although there is still much to be understood, these simulations already show some of our theoretical predictions and are useful to form a global picture of the process. Additional details will be published elsewhere. Figure 5 compares the light-spring effects near the plasma cutoff in the linear and in the nonlinear regime. The simulation details are identical to those in Figure 4, except for the laser peak vector potential, which in the nonlinear regime is $a_{1}=a_{2}=1.7$, i.e., the peak $a_{0}$ for the combined modes is close to $a_{1}+a_{2}=3.4$. Figure $5 \mathrm{a}$ illustrates the electric field of a light spring laser pulse in the linear regime, where the peak vector potential $a_{0}$ is below unity. Here, the laser pulse is uniformly reflected along the transverse $x_{2}$ direction and at the longitudinal position that corresponds to the critical density. In contrast, simulations in the nonlinear regime, shown in Figure 5b), show that the laser pulse profile near the reflection position is not symmetric around the $x_{2}=0$ axis. The laser pulse penetrates deeper into the target for $x_{2}>0$ than for $x_{2}<0$. Furthermore, the longitudinal reflection point $x_{2}$ is beyond the critical surface. This asymmetry is also consistent in Figure 5c), which shows the corresponding plasma electron density. In addition to relativistic self-induced transparency, these results also suggest that the holeboring process may follow the helical structure of the light spring (to be further explored in a separate publication). 
a)

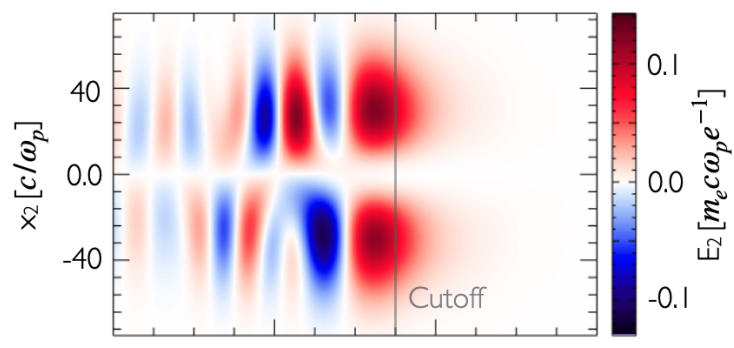

b)

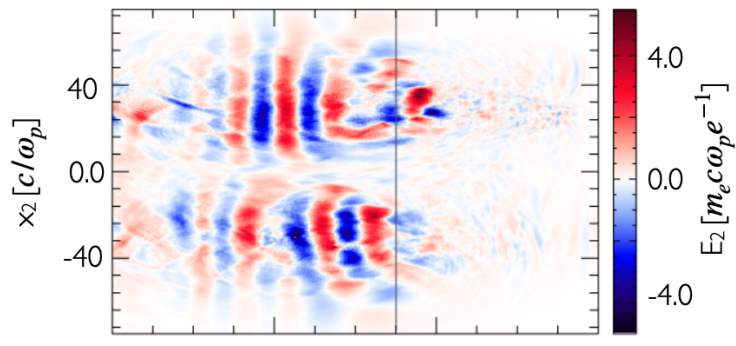

c)

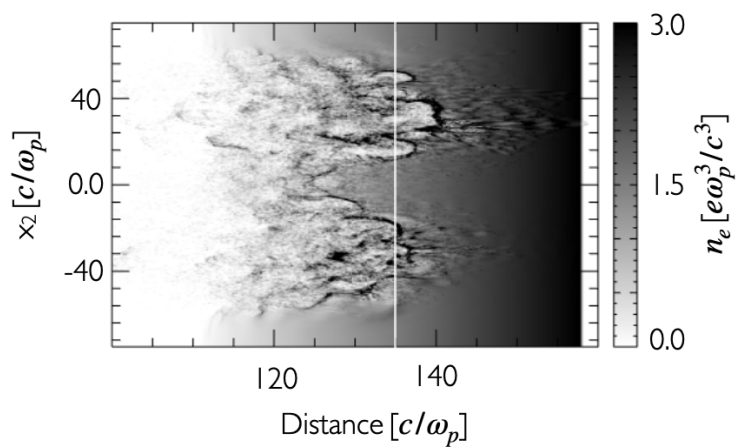

Figure 5. Simulation results of a light spring near plasma cutoff in the linear regime in (a) and in the nonlinear regime in (b), and of the electron density during the light-spring interaction (nonlinear) in (c), confirming the analytical predictions of self-induced transparency and hole boring, respectively. The OSIRIS code was used.

\section{Conclusions}

In this work, we have considered propagation of twisted waves in inhomogeneous and unmagnetised plasma. We discussed the main wave properties in the cutoff region and determined the electric field solutions. We also examined the qualitative differences between reflection of a simple twisted wave, described by a single Laguerre-Gauss (LG) mode, and reflection of a light spring, described by a superposition of different LG modes. In particular, we have shown that a light spring indeed behaves as a real (mechanical) spring near cutoff, because it contracts when it moves closer to reflection, and expands after reflection as a mechanical spring would do after being released of compression.

Our aim here was to understand the main qualitative changes associated with twisted waves, or in other words, with waves carrying orbital angular momentum. For this reason, we mainly considered wave propagation in the linear regime. This allowed us to discover surprising new aspects of twisted waves near plasma cutoff. The most relevant one, in the linear regime, is the light-spring effect, which corresponds to wave-packet contraction and expansion in the cutoff region, strongly resembling a mechanical spring.

The nonlinear properties were also analysed. We have shown, with the help of a simple analytical model and particle-in-cell simulations, that in the weakly relativistic regime two distinct processes could be identified: self-induced transparency and hole boring. Some evidence of excitation of low frequency oscillations, eventually excited by the fast electron beam moving into the overdense region, were also found. Self-induced transparency is a well-known consequence of the relativistic electron response to the incident laser field [20-22]. Hole boring has been examined in the context of Gaussian beams, and is a 
subject of current interest [23-25]. These processes are modified here due to the particular field configurations associated with twisted waves. However, we also observed really new processes, which are not apparent in Gaussian beams, such as the generation of helical holes and axial quasi-static $B$ fields.

Here, we focused on the case of normal incidence. It is well known that for incident plane waves at oblique incidence, the two polarisation states (parallel and perpendicular to the incidence plane) have distinct qualitative features. This means that, even for linear propagation, the exchange of angular momentum between the wave and the medium will eventually be more complicated. For that reason, oblique incidence will be examined in a separate publication.

Author Contributions: Conceptualization, J.T.M., C.W. and J.V.; methodology, J.T.M., C.W. and J.V.; software, J.T.M., C.W. and J.V.; validation, J.T.M., C.W. and J.V.; formal analysis, J.T.M., C.W. and J.V.; investigation, J.T.M., C.W. and J.V.; resources, J.T.M., C.W. and J.V.; data curation, J.T.M., C.W. and J.V.; writing-original draft preparation, J.T.M., C.W. and J.V.; writing-review and editing, J.T.M., C.W. and J.V.; visualization, J.T.M., C.W. and J.V.; supervision, J.T.M., C.W. and J.V.; project administration, J.T.M., C.W. and J.V.; funding acquisition, J.T.M., C.W. and J.V. All authors have read and agreed to the published version of the manuscript.

Funding: This research was partly funded by Fundação para a Ciência e Tecnologia (Portugal), grant number SFRH/IF/01635/2015.

Data Availability Statement: The data presented in this study are included in the Figures.

Acknowledgments: One of the authors (J.V.) would like to acknowledge the support of FCT (Portugal) Grant No. SFRH/IF/01635/2015.

Conflicts of Interest: The authors declare no conflict of interest.

\section{References}

1. Budden, K.G. Radio Waves in the Ionosphere; Cambridge University Press: London, UK, 1961.

2. Ginzburg, V.L. Propagation of Electromagnetic Waves in Plasmas; Pergamon Press: New York, NY, USA, 1964.

3. White, R.B.; Chen, F.F. Amplification and absorption of electromagnetic waves in overdense plasmas. Plasma Phys. 1974, 16, 565. [CrossRef]

4. Stenzel, R.L.; Wong, A.Y.; Kim, H.C. Conversion of electromagnetic waves to electrostatic waves in inhomogeneous plasmas. Phys. Rev. Lett. 1974, 32, 654. [CrossRef]

5. Freidberg, J.P.; Mitchell, R.W.; Morse, R.L.; Rudsinski, L.I. Resonant absorption of laser light by plasma targets. Phys. Rev. Lett. 1972, 28, 795. [CrossRef]

6. Kolodner, P.; Yablonovitch, E. Two-dimensional distribution of self-generated magnetic fields near the laser-plasma resonantinteraction region. Phys. Rev. Lett. 1979, 43, 1402. [CrossRef]

7. Dragila, R.; Krepelka, J. Laser plasma density profile modification by ponderomotive force. J. Physique 1978, 39, 617-623. [CrossRef]

8. Yao, A.M.; Padgett, M.J. Orbital angular momentum: Origins, behavior and applications. Adv. Opt. Photonics 2011, 3, 161-204. [CrossRef]

9. Secor, J.; Alfan, R.; Ashrafi, S. Complex Light; Institute of Phys. Publishing: Bristol, UK, 2017.

10. Mendonça, J.T. Twisted waves in a plasma. Plasma Phys. Control. Fusion 2012, 54, 124031. [CrossRef]

11. Mendonça, J.T.; Thidé, B.; Then, H. Stimulated Raman and Brillouin backscattering of collimated beams carrying orbital angular momentum. Phys. Rev. Lett. 2009, 102, 185005. [CrossRef]

12. Vieira, J.; Trines, R.M.G.M.; Alves, E.P.; Fonseca, R.A.; Mendonça, J.T.; Bingham, R.; Norreys, P.; Silva, L.O. Amplification and generation of ultra-intense twisted laser pulses via stimulated Raman scattering. Nat. Commun. 2016, 7, 10371. [CrossRef] [PubMed]

13. Vieira, J.; Mendonça, J.T.; Quéré, F. Optical control of the topology of laser-plasma accelerators. Phys. Rev. Lett. 2018, 121, 054801. [CrossRef] [PubMed]

14. Pariente, G.; Quéré, F. Spatio-temporal light springs: Extended encoding of orbital angular momentum in ultrashort pulses. Opt. Lett. 2015, 40, 2037-2040. [CrossRef] [PubMed]

15. Vieira, J.; Mendonça, J.T. Nonlinear laser driven donut wakefields for positron and electron acceleration. Phys. Rev. Lett. 2014, 112, 215001. [CrossRef]

16. Abramowitz, M.; Stegun, I.A. (Eds.) Handbook of Mathematical Functions with Formulas, Graphs, and Mathematical Tables; NBS Applied Mathematics Series 55; National Bureau of Standards: Washington DC, USA, 1964. 
17. Fonseca, R.A.; Silva, L.O.; Tsung, F.S.; Decyk, V.K.; Lu, W.; Ren, C.; Mori, W.B.; Deng, S.; Lee, S.; Katsouleas, T.; et al. OSIRIS: A Three-dimensional, Fully Relativistic Particle in Cell Code for Modelling Plasma Based Accelerators. In Lecture Notes in Computer Science, Proceedings of the International Conference on Computational Science, Amsterdam, The Netherlands, 21-24 April 2002; Springer: Berlin/Heidelberg, Germany, 2002; Volume 2331, pp. 342-351.

18. Fonseca, R.A.; Vieira, J.; Fiuza, F.; Davidson, A.; Tsung, F.S.; Mori, W.B.; Silva, L.O. Exploiting multi-scale parallelism for large scale numerical modelling of laser wakefield accelerators. Plasma Phys. Controlled Fusion 2013, 55, 124011. [CrossRef]

19. Mendonça, J.T.; Vieira, J. High harmonic generation in underdense plasmas by intense laser pulses with orbital angular momentum. Phys. Plasmas 2015, 22, 123106. [CrossRef]

20. Akhiezer, A.I.; Polovin, R.V. Theory of wave motion of an electron plasma. Sov. Phys. JETP 1956, 3, 696.

21. Kaw, P.; Dawson, J. Relativistic nonlinear propagation of laser beams in cold overdense plasmas. Phys. Fluids 1970, $13,472-481$. [CrossRef]

22. Max, C.; Perkins, F. Strong electromagnetic waves in overdense plasmas. Phys. Rev. Lett. 1971, 27, 1342. [CrossRef]

23. Iwata, N.; Kojima, S.; Sentoku, Y.; Hata, M.; Mima, K. Plasma density limits for hole boring by intense laser pulses. Nat. Commun. 2018, 9, 623. [CrossRef]

24. Hornung, J.; Zobus, Y.; Roeder, S.; Kleinschmidt, A.; Bertini, D.; Zepf, M.; Bagnoud, V. Time-resolved study of hole boring in realistic experimental conditions. Nat Commun. 2021, 12, 6999. [CrossRef] [PubMed]

25. Weng, S.M.; Shenga, Z.M.; Murakamid, M.; Chena, M.; Liua, M.; Wanga, H.C.; Yuana, T.; Zhang, J. Optimization of hole-boring radiation pressure acceleration of ion beams for fusion ignition. Matter Radiat. Extrem. 2018, 3, 28-39. [CrossRef] 